\title{
Ablação por Cateter de Taquicardia Atrial Focal com Ativação Precoce Próxima ao Feixe de His, a Partir da Cúspide Aórtica não Coronária
}

\author{
Catheter Ablation of Focal Atrial Tachycardia with Early Activation Close to the His-Bundle from the Non \\ Coronary Aortic Cusp \\ Muhieddine Chokr, ${ }^{1}{ }^{\circledR 0}$ Lucas G. de Moura, ${ }^{1}{ }^{[0}$ Italo Bruno dos Santos Sousa, ${ }^{1}$ Cristiano Faria Pisani, ${ }^{10}$ Carina \\ Abigail Hardy, ${ }^{1}$ Sissy Lara de Melo, ${ }^{1 \oplus}$ Arnobio Dias da Ponte Filho, ${ }^{2}{ }^{\circledR}$ leda Prata Costa, ${ }^{\circledR}$ Ronaldo Vasconcelos \\ Tavora, ${ }^{2}$ Luciana Sacilotto, ${ }^{10}$ Tan Chen Wu, ${ }^{10}$ Francisco Carlos da Costa Darrieux, ${ }^{1}$ Denise Tessariol Hachul, ${ }^{1}$ Vera \\ Demarchi Aiello, ${ }^{10}$ Mauricio Scanavacca ${ }^{10}$ \\ Universidade de São Paulo Faculdade de Medicina Hospital das Clinicas Instituto do Coração, ${ }^{1}$ São Paulo, SP - Brasil \\ Antonio Prudente Hospital, ${ }^{2}$ Fortaleza, CE - Brasil
}

\section{Resumo}

Fundamento: A ablação da taquicardia atrial (TA) com local de ativação mais precoce próxima ao feixe de His é um desafio, devido ao risco de bloqueio de AV completo por sua proximidade ao sistema de His-Purkinje (SHP). Uma alternativa para minimizar esse risco é posicionar o cateter na cúspide não coronária $(\mathrm{CNC})$, que é anatomicamente contígua à região para-Hissiana.

Objetivos: O objetivo deste estudo foi fazer uma revisão de literatura e avaliar as características eletrofisiológicas, a segurança e o índice de sucesso de aplicação de radiofrequência (RF) por cateter na CNC para o tratamento de TA paraHissiana em uma série de casos.

Métodos: Avaliamos retrospectivamente dez pacientes (Idade: 36 \pm 10 anos) que foram encaminhados para ablação de taquicardia paroxística supraventricular (TPSV) e haviam sido diagnosticados com TA focal para-Hissiana confirmada por manobras eletrofisiológicas clássicas. Para a análise estatística, um $P$ valor $\mathbf{d}<0.05$ foi considerado estatisticamente significativo.

Resultados: A ativação atrial mais precoce na posição His foi de $28 \pm 12 \mathrm{~ms}$ da onda $\mathrm{P}$, e a CNC foi $3 \pm 2 \mathrm{~ms}$ antes da posição His, sem evidência de potencial His em todos os pacientes. Foi aplicada RF à CNC (cateter de ponta de 4-mm; 30W, $55^{\circ} \mathrm{C}$ ) e a taquicardia foi interrompida em $5 \pm 3$ s sem aumento no intervalo PR ou evidência de um ritmo juncional. Os testes eletrofisiológicos não induziram novamente a taquicardia em 9/10 pacientes. Não houve complicações em nenhum procedimento. Durante o período de acompanhamento de $30 \pm 12$ meses, nenhum paciente apresentou recorrência de taquicardia.

Conclusão: O tratamento percutâneo de TA para-Hissiana por meio de CNC é uma estratégia segura e eficiente, tornandose uma opção interessante para o tratamento de arritmia complexa. (Arq Bras Cardiol. 2021; 116(1):119-126)

Palavras-chave: Arritmias Cardíacas; Taquicardia Atrial; Ablação por Cateter/métodos; Fascículo Atrioventricular; Técnicas Eletrofisiológicas Cardíacas/métodos; Eletrocardiografia/métodos.

\footnotetext{
Abstract

Background: Atrial tachycardia (AT) ablation with earliest activation site close to the His-Bundle is a challenge due to the risk of complete AV block by its proximity to His-Purkinje system (HPS). An alternative to minimize this risk is to position the catheter on the non-coronary cusp (NCC), which is anatomically contiguous to the para-Hisian region.

Objectives: The aim of this study was to perform a literature review and evaluate the electrophysiological characteristics, safety, and success rate of catheter-based radiofrequency (RF) delivery in the NCC for the treatment of para-Hisian AT in a case series.

Methods: This study performed a retrospective evaluation of ten patients (Age: $36 \pm 10$-o) who had been referred for SVT ablation and presented a diagnosis of para-Hisian focal AT confirmed by classical electrophysiological maneuvers. For statistical analysis, a p-value of <0.05 was considered statistically significant.

Correspondência: Muhieddine Chokr •

Universidade de São Paulo Faculdade de Medicina Hospital das Clinicas Instituto do Coração - Unidade de arritmias cardíacas - Av. Dr. Enéas Carvalho de

Aguiar, 44. CEP 05403-900, Cerqueira Cesar, São Paulo, SP - Brasil

E-mail: muhieddinechokr@hotmail.com

Artigo recebido em 27/02/2019, revisado em 14/11/2019, aceito em 27/12/2019
}

DOI: https://doi.org/10.36660/abc.20180449 
Results: The earliest atrial activation at the His position was $28 \pm 12 \mathrm{~ms}$ from the $P$ wave and at the NCC was $3 \pm 2 m s$ earlier than His position, without evidence of His potential in all patients. RF was applied on the NCC (4-mm-tip catheter; 30W, 55 $\left.{ }^{\circ} \mathrm{C}\right)$, and the tachycardia was interrupted in $5 \pm 3 s$ with no increase in the $P R$ interval or evidence of junctional rhythm. Electrophysiological tests did not reinduce tachycardia in 9/10 of patients. There were no complications in all procedures. During the $30 \pm 12$ months follow-up, no patient presented tachycardia recurrence.

Conclusion: The percutaneous treatment of para-Hisian AT through the NCC is an effective and safe strategy, which represents an interesting option for the treatment of this complex arrhythmia. (Arq Bras Cardiol. 2021; 116(1):119-126)

Keywords: Arrhythmias, Cardiac; Tachycardia, Atrial; Catheter, Ablation/methods; Bundle of His; Electrophysiologic,Techniques/methods; Electrocardiography/methods.

Full texts in English - http://www.arquivosonline.com.br

\section{Introdução}

As taquicardias atriais focais (TA) geralmente se originam de determinadas estruturas compostas de tecido atrial, tais como a crista terminalis, veias pulmonares, apêndices atriais e óstio do seio coronário. A ablação por cateter com radiofrequência (RF) foi estabelecida como o método preferencial para o tratamento dessas arritmias. Embora focos originados na região para-Hissiana sejam raros, eles são um desafio terapêutico devido à proximidade com o sistema de His-Purkinje (SHP). A tentativa de ablação via átrio direito poderia aumentar o risco de afetar o nó $\mathrm{AV}$, SHP, e, portanto, causar o bloqueio atrioventricular (AV). Entretanto, o uso do acesso retroaórtico para explorar a cúspide não coronária $(\mathrm{CNC})$, que é anatomicamente adjacente à região mencionada acima, é descrito como uma estratégia alternativa. ${ }^{1}$ A experiência com a eficácia e a segurança desse tipo de ablação continua limitada. Neste estudo, relatamos uma série de casos de taquicardia atrial para-Hissiana que foram mapeados e fizeram a ablação pela CNC. As características eletrofisiológicas e os resultados com essa abordagem foram analisados. Além disso, a anatomia da região e as estratégias procedimentais foram discutidas.

\section{Método}

Foram analisados os prontuários de 10 pacientes (8 mulheres e 2 homens; média de idade 36 110 anos), de duas instituições brasileiras (Instituto do Coração/InCor, Faculdade de Medicina da Universidade de São Paulo. e Hospital Antônio Prudente, Fortaleza), submetidos a ablação por cateter entre janeiro de 2014 e março de 2017. O tratamento com drogas antiarrítmicas foi interrompido por pelo menos cinco meias-vidas antes do procedimento. Eles foram avaliados por exame físico, radiografia torácica, e ecocardiograma, e nenhum apresentou doença cardíaca estrutural.

Os pacientes foram submetidos a um estudo eletrofisiológico após jejum de 8 horas, sob monitoramento contínuo e nível de sedação controlado por um anestesista. Foi realizada punção tripla na veia femoral, e cateteres padrão (3) foram introduzidos no seio coronário (decapolar; $6 F$ ), região do feixe His (quadripolar, 7F), e base do ventrículo direito (quadripolar, 7F).

A estimulação atrial programada, ou ruptura atrial foi feita com um sistema de registro de eletrofisiologia
(EP tracer, Holanda) para induzir a taquicardia em dois pacientes; e o aparecimento espontâneo de taquicardia foi observado em um paciente. O uso de isoproterenol (10-20mcg; infusão IV) foi necessário em sete pacientes. Em um caso, estava disponível um sistema de mapeamento eletroanatômico (Carto 3; Biosense).

O diagnóstico de TA foi confirmado utilizando-se as seguintes observações e manobras eletrofisiológicas: alterações no intervalo A-A antes de alterações no intervalo $\mathrm{V}-\mathrm{V}$, arrastamento ventricular durante a taquicardia com resposta tipo $\mathrm{V}-\mathrm{A}-\mathrm{A}-\mathrm{V}$, ou mesmo alterações no intervalo V-A durante a taquicardia (ausência de conexão V-A). Em todos os casos, observou-se a ativação atrial com menos de $50 \%$ do comprimento do ciclo de taquicardia, indicando um padrão de ativação focal.

Quando a ativação atrial mais precoce estava no septo atrial direito e era acompanhada de potencial His perceptível no local, a TA foi definida como para-Hissiana. Por último, a artéria femoral foi puncionada para permitir o mapeamento detalhado da região da válvula aórtica retrógrada.

Um cateter terapêutico de ponta de 4-mm foi usado para aplicação de radiofrequência (RF) (30W/55ํC durante 60 segundos), usando incidências fluoroscópicas oblíquas direita e esquerda como referências para a localização anatômica (Figura 1). Em um paciente, foi usado um sistema de mapeamento eletroanatômico (Figura 2). Em todos os casos, o local da ativação mais precoce foi identificado pela $\mathrm{CNC}$, em relação ao aparecimento da onda $\mathrm{P}$ periférica, semelhante ao detectado pelo cateter colocado no septo interatrial direito, mas com a vantagem da ausência do potencial de feixe de His no primeiro (Figura 3). O sucesso do procedimento foi definido como sendo a cessação da taquicardia durante a aplicação de RF, e a não indução da taquicardia depois de várias tentativas de induzi-la por ruptura atrial, ou depois da infusão de isoproterenol.

\section{Análise estatística}

Os dados contínuos são apresentados como média \pm desvio padrão (DP), se forem normalmente distribuídos, e como mediana mais faixa interquartil, se não forem. Para dados categóricos, serão usadas contagens e porcentagens (\%). O teste de Shapiro-Wilk foi usado para determinar a normalidade da distribuição. O teste $U$ de Mann Whitney foi utilizado para comparar diferenças entre grupos para valores contínuos não paramétricos. Por último, o teste exato de Fisher foi aplicado para avaliação de dados categóricos em 


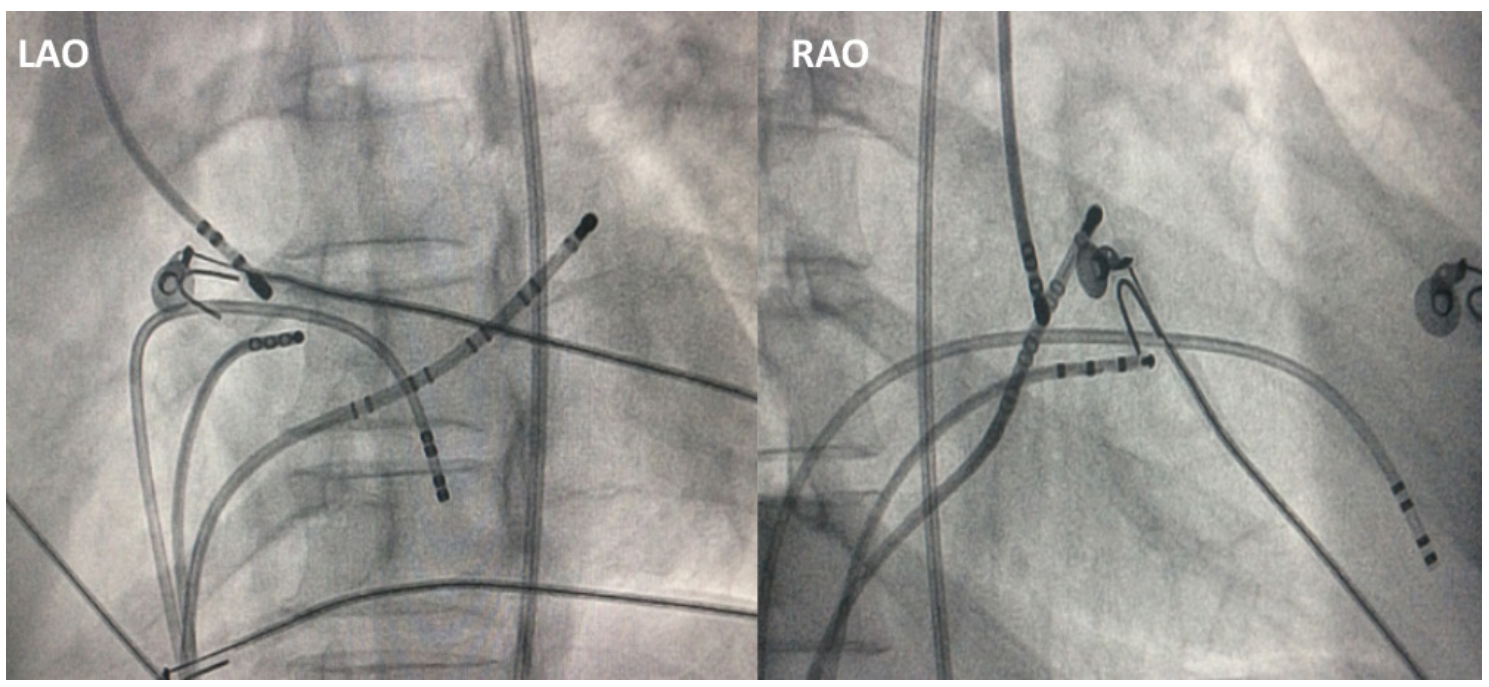

Figura 1 - Posicionamento do cateter de ablação na CNC em projeção obliqua direita e esquerda.

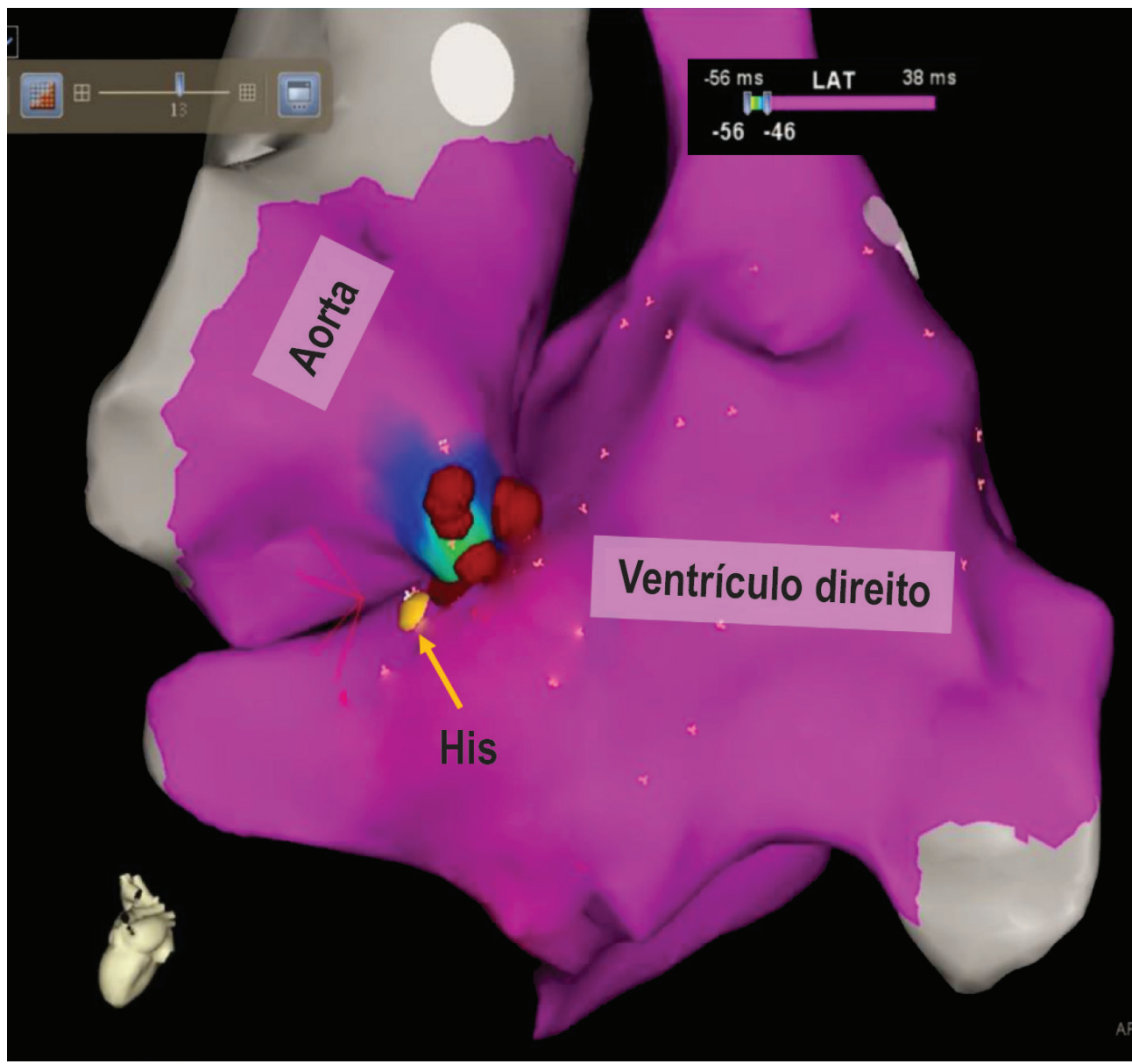

Figura 2 - Mapeamento eletroanatômico mostrando pontos de aplicação de RF na CNC. Observe o relacionamento íntimo da cúspide não coronária com a região para-Hissiana. 


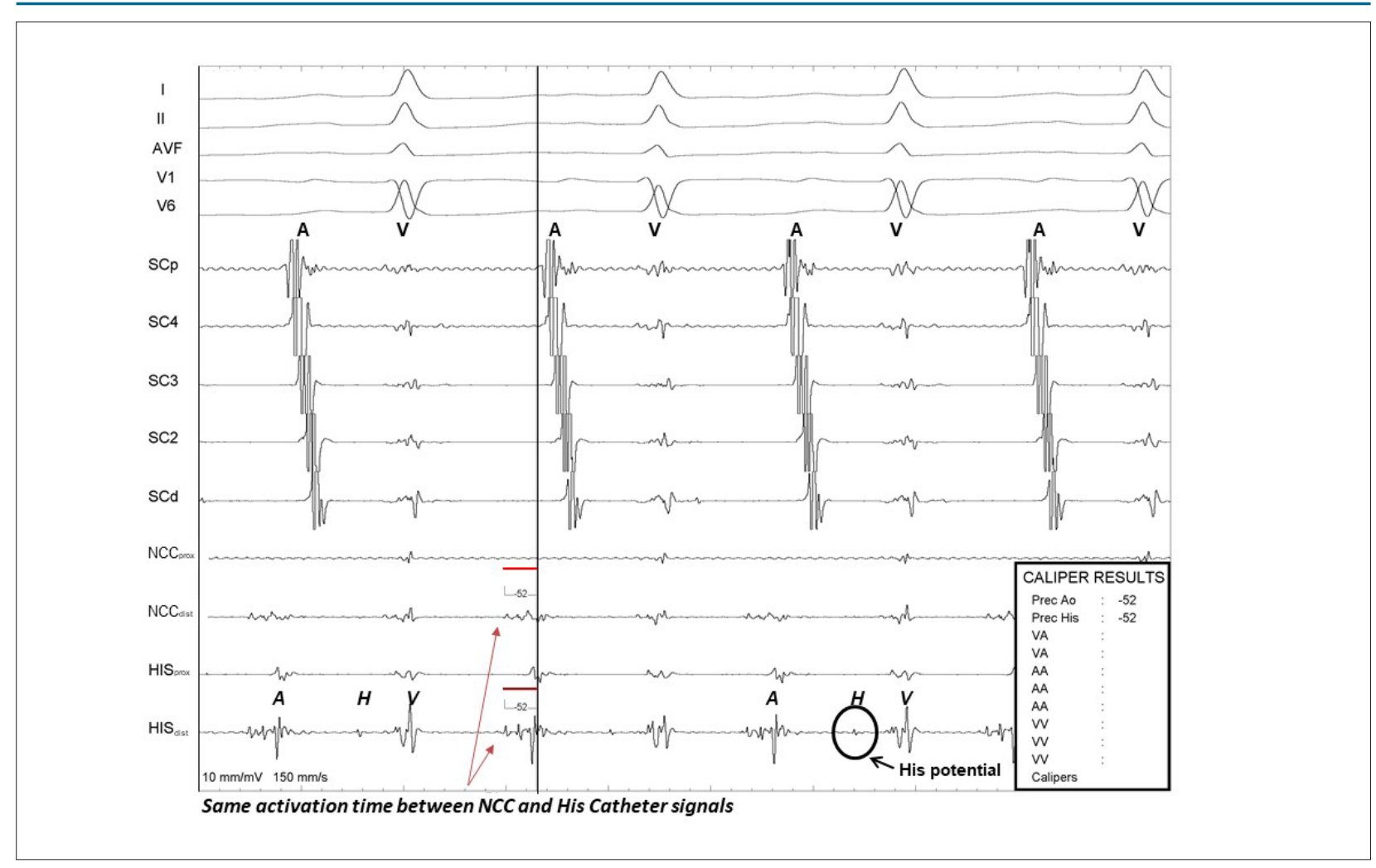

Figura 3 - Em sequência, de cima para baixo: derivações periféricas, seio coronário de eletrodos proximais e distais, e registro de cateteres próximos à região da válvula aórtica e do feixe de His. Tempo de ativação atrial local mais precoce semelhante é observado em relação ao aparecimento de uma onda $P$ da região septal para-Hissiana e CNC, mas não se observou potencial His nesta última.

uma tabela de contingência $2 \times 2$. Para todos os testes, um P valor $\mathrm{d}<0,05$ foi considerado estatisticamente significativo (bilateral). A análise estatística foi realizada com o software SPSS, versão 19.0 (SPSS Inc., Chicago, IL).

\section{Resultados}

As características clínicas e eletrofisiológicas dos pacientes podem ser visualizadas na Tabela 1 e na Tabela 2. Todas as variáveis contínuas, exceto a duração da onda $\mathrm{P}$ durante o ritmo do seio e a taquicardia, apresentaram distribuição normal (Tabela 2).

Nenhum dos pacientes já tinha passado por ablação por cateter. O comprimento médio do ciclo de taquicardia atrial foi $362 \pm 43 \mathrm{~ms}$. Ativação atrial mais precoce registrada no cateter His foi $28 \pm 12 \mathrm{~ms}$, em relação à onda $\mathrm{P}$ periférica. $\mathrm{O}$ tempo de ativação atrial local registrado pelo cateter na CNC foi $3 \pm 2$ ms antes, comparado ao do cateter His.

Em todos os casos, o local inicial de ablação foi a $\mathrm{CNC}$, com sucesso em 9 de 10 deles. O caso restante exigiu o mapeamento e a tentativa de ablação na região para-Hissiana com baixa potência (20w), que também não foi bem sucedido.

O tempo médio para a interrupção da taquicardia atrial depois da aplicação de RF foi 5 segundos. O ritmo juncional e um aumento no intervalo PR não foram observados durante a aplicação de RF em todos os casos. Todos os procedimentos foram bem tolerados e não houve complicações.
Durante um período de acompanhamento de $30 \pm 12$ meses, nenhum paciente teve recorrência de taquicardia atrial. Os pacientes permaneceram assintomáticos na avaliação clínica e com monitoramento por ECG Holter contínuo dinâmico.

Ao registrar o eletrocardiograma de superfície, observamos que a morfologia da onda $\mathrm{P}$, demonstrando padrão bifásico ou trifásico em 6 de 10 pacientes em derivações inferiores, e teve duração significativamente mais curta, em comparação com o ritmo do seio em todos os casos, $93 \pm 17$ versus 112 $\pm 20 \mathrm{~ms}(\mathrm{p}<0,05)$. (Figura 4).

\section{Discussão}

\section{Relações morfológicas}

A aorta ocupa uma posição central na base do coração, encaixada profundamente entre as junções atrioventriculares direita e esquerda. As relações espaciais dos seios de Valsalva demonstram, portanto, proximidade entre as paredes atriais e o tecido adiposo interposto entre eles na base do coração. Considerando um padrão de meia-lua da fixação dos componentes aórticos, é evidente que as relações topográficas variam de acordo com a profundidade dentro do seio. A Figura 5A e a Figura 5B mostram as relações anatômicas do seio aórtico não coronário em relação às estruturas do átrio direito. Especificamente, a parte mais profunda do seio aórtico não 


\begin{tabular}{|c|c|c|c|c|c|c|}
\hline Paciente & Idade & Sexo & $\begin{array}{c}\text { Doença cardíaca } \\
\text { estrutural }\end{array}$ & Duração dos sintomas (meses) & AADs ineficazes & $\begin{array}{c}\text { Ablação anterior } \\
\text { mal sucedida }\end{array}$ \\
\hline 1 & 38 & $\mathrm{~F}$ & Nenhuma & $\leq 12$ & 0 & Não \\
\hline 2 & 49 & M & Nenhuma & $12-24$ & 2 & Não \\
\hline 3 & 22 & $\mathrm{~F}$ & Nenhuma & $12-24$ & 1 & Não \\
\hline 4 & 28 & $\mathrm{~F}$ & Nenhuma & $12-24$ & 0 & Não \\
\hline 5 & 31 & $\mathrm{~F}$ & Nenhuma & $\geq 48$ & 3 & Não \\
\hline 6 & 33 & $\mathrm{~F}$ & Nenhuma & $\geq 48$ & 1 & Não \\
\hline 7 & 46 & $\mathrm{~F}$ & Nenhuma & $\leq 12$ & 1 & Não \\
\hline 8 & 58 & $\mathrm{~F}$ & Nenhuma & $12-24$ & 2 & Não \\
\hline 10 & 30 & $\mathrm{~F}$ & Nenhuma & $\leq 12$ & 1 & Não \\
\hline
\end{tabular}

Tabela 2 - Características eletrofisiológicas dos pacientes avaliados

\begin{tabular}{|c|c|}
\hline Variável & Valor \\
\hline Média de idade (anos) & $36 \pm 10^{*}$ \\
\hline Duração da onda p durante a taquicardia (ms) & $93 \pm 17^{\S}$ \\
\hline Duração da onda p durante o ritmo do seio (ms) & $112 \pm 20 \S$ \\
\hline Índice de sucesso da ablação & $9 / 10(90 \%)$ \\
\hline Comprimento médio do ciclo de taquicardia (ms) & $362 \pm 43^{*}$ \\
\hline Ativação atrial mais precoce registrada no cateter His (ms) & $28 \pm 12^{*}$ \\
\hline Local da ativação mais precoce à região do feixe de His (ms) & $3 \pm 2^{*}$ \\
\hline Tempo médio de fluoroscopia (minutos) & $14[10-18]$ \\
\hline Tempo do início da ablação até a interrupção da taquicardia (segundos) & $5[2-8]$ \\
\hline Uso de sistema de mapeamento 3D & $1 / 10$ \\
\hline Angiografia coronária & $0 / 10$ \\
\hline Complicações graves & $0 / 10$ \\
\hline
\end{tabular}

*Os dados são apresentados como média + desvio padrão (DP). §O valor é exibido como média + faixa interquartil (IQR).

coronário (também chamado não adjacente) está intimamente relacionada ao componente atrioventricular do septo cardíaco. A Figura 5C mostra a parede do seio e os marcos da área juncional e nó atrioventricular

Dessa forma, a CNC passa a ser um alvo alternativo na estratégia terapêutica em que a falha na intervenção ocorre quando se tenta fazer a ablação nos dois lados do septo atrial, ou o risco de bloqueio atrioventricular resulta do registro de eletrograma do feixe de His próximo ao alvo da ablação. ${ }^{2}$

Do ponto de vista embriológico, células da crista neural contribuem para formar o septo aortopulmonar, coxim endocárdico no canal de saída, e isolamento do feixe de His do miocárdio ao seu redor. Resquícios dessas células na região perinodal pode justificar o substrato, que causa e mantém a arritmia. ${ }^{3}$ A CNC se origina do miocárdio atrial, enquanto a cúspide coronária esquerda se origina do miocárdio ventricular. Esse fato explica a frequência de arritmias atriais na NCC, e arritmias ventriculares nas cúspides direita e esquerda. ${ }^{3}$
A prevalência de taquicardias originárias da região perinodal é de 7 a 10\% em séries diferentes, com várias séries e relatos de casos mostrando que taquicardias para-Hissianas podem ser tratadas adequadamente, com um índice de complicação baixo. ${ }^{4}$

A abordagem dessas taquicardias através da $\mathrm{CNC}$ reduz o risco de danos ao sistema de condução, garantindo uma estabilidade maior ao cateter durante a aplicação de RF, bem como um bom contato com o tecido. A explicação provável para a eficiência da ablação nesse local é o direcionamento das extensões atriais direitas na $\mathrm{CNC}$, mais distante do sistema His Purkinje, que se localiza no endocárdio. ${ }^{5}$

Em relação a complicações, a aplicação de RF pode causar danos às válvulas cardíacas, embora essa complicação ainda não foi relatada até os limites de potência (30w) e temperatura $\left(55^{\circ} \mathrm{C}\right)$ em várias séries. ${ }^{6} \mathrm{~A}$ angiografia coronária não foi realizada como rotina antes de se aplicar a RF porque nossa prática demonstra que a presença de um eletrograma com átrio maior que o ventrículo (razão A/V > 1), anatomicamente 


\section{Artigo Original}

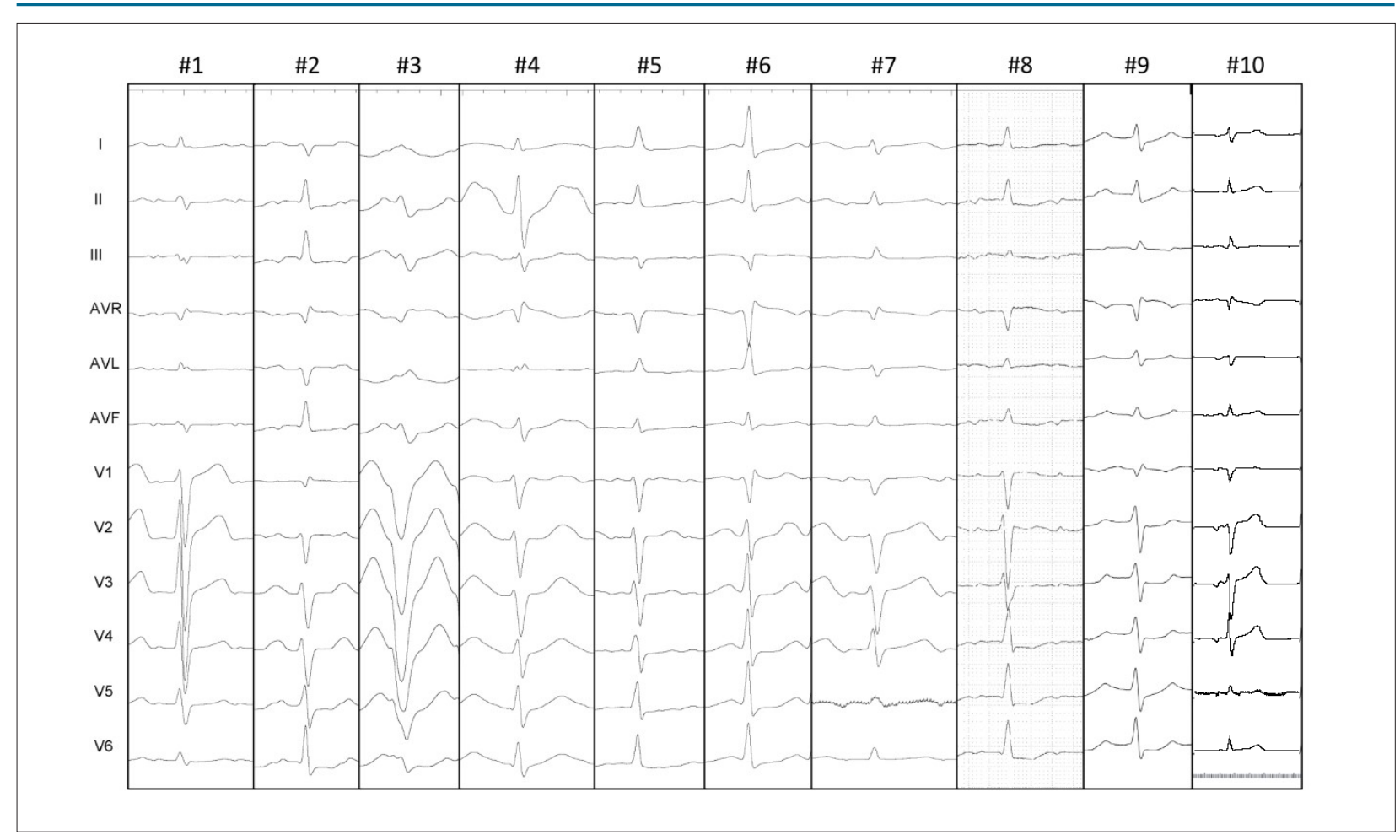

Figura 4 - Morfologia da onda $P$ de todos os casos. Morfologia da onda $P$, demonstrando padrão bifásico ou trifásico em 6 de 10 pacientes em derivações inferiores.
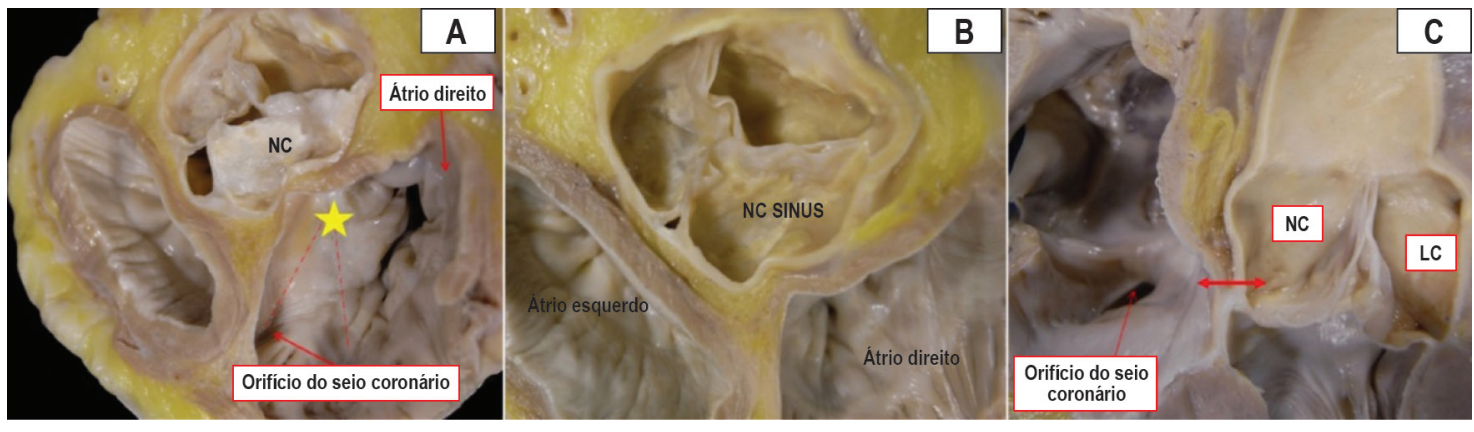

Figura 5 - Pode-se observar uma relação íntima entre o CNC e a região do feixe de His. A) Vista oblíqua de um corte de eixo curto na base do coração, mostrando o seio não coronário de Valsalva (NC) e os marcos do triângulo de Koch (linhas pontilhadas) e septo membranoso (estrela). B) Corte de eixo curto na base do coração mostrando as relações espaciais dos seios aórticos e o tecido adiposo presente entre a aorta e as paredes atriais. C) Seção longitudinal da raiz aórtica mostrando a curta distância entre a porção profunda do seio não coronário de Valsalva (CNC) e a área correspondente ao vértice do triângulo de Koch, localizado na região anterossuperior em relação ao orifício do seio coronário (seta com duas pontas).

mais próximo a um cateter usado como referência no átrio direito, paralelo ao sistema de condução, marca um local seguro para a ablação. Em relação à técnica de mapeamento, observou-se uma razão maior ou igual a 1 entre as amplitudes dos eletrogramas atrial e ventricular em todos os pacientes do alvo de ablação. Essa característica eletrofisiológica tem grande valor, pois a inversão da relação $\mathrm{A} / \mathrm{V}$ sugere que o limite da CNC está sendo ultrapassado e o cateter está apoiado na cúspide direita. Isso causa maior risco de lesão ao sistema de condução, servindo como assistência e referência anatômica quando a fluoroscopia é usada.
Usamos o mapeamento eletroanatômico (MEA) apenas em um caso. O motivo disso é que a maioria de nossos pacientes foram tratados no sistema público de saúde, onde esse recurso não está disponível. Entretanto, em nossa amostra, conforme descrito por Toniolo et al., ${ }^{8}$ foi possível alcançar altos índices de sucesso, apesar de não usar o recurso.

Por outro lado, há situações em que o MEA é fundamental. Recentemente, Bitaraes et al publicaram um caso de uma paciente grávida com uma TA refratária a tratamento farmacológico, em que a ablação por cateter foi realizada 
com sucesso pela cúspide aórtica não coronária com fluoroscopia zero, utilizando o MEA. ${ }^{9}$

Nossos achados estão em desacordo com os de Ouyang et al., ${ }^{10}$ que observaram que uma onda $\mathrm{P}-/+$ na derivação V1 associada a P+ em D1 e AVL sugerem origem na CNC. De acordo com esse autor, a relação entre a presença de uma onda $\mathrm{P}-/+$ com sua parte mais proeminente sendo positiva e a origem no átrio esquerdo é um fato relevante. Recentemente, Madaffari et al. ${ }^{11}$ publicaram dados da morfologia da onda $\mathrm{P}$, em que uma onda P estreita e bifásica $(-/+)$ ou trifásica $(+/-/+)$ nas derivações inferior e precordial identificam com confiança o grupo de TA que surge na região para-Hissiana. Em nosso estudo, identificamos que a morfologia da onda $\mathrm{P}$, demonstrando padrão bifásico ou trifásico em 6 de 10 pacientes em derivações inferiores, e uma onda $\mathrm{P}$ significativamente mais curta em comparação com o ritmo do seio, era variável nas derivações precordiais.

Em nosso estudo, em dez casos, ocorreu apenas uma tentativa mal sucedida de realizar a ablação de taquicardia pela $\mathrm{CNC}$, que também não teve sucesso pelo átrio direito. Presumimos que uma estratégia mais agressiva pelo lado direito do septo poderia ter resultado em danos ao sistema de condução e bloqueio atrioventricular, justificando a baixa potência de saída testada (20w). A taquicardia parou durante as aplicações, mas pode ser induzida novamente durante a infusão de isoproterenol. Um alvo mais profundo na região do septo poderia explicar a dificuldade de eliminar o substrato. Outra limitação é que o operador não explorou a região esquerda do septo nesse caso. Também não usamos cateter irrigado porque, em nossa opinião, a raiz aórtica é uma região de alto fluxo de sangue e, a menos que a aplicação da potência fosse limitada por cortes de alta temperatura repetidamente, a irrigação não deveria fazer uma diferença significativa. No acompanhamento clínico, o paciente estava assintomático, com o uso de betabloqueadores. Portanto, nenhuma nova tentativa de ablação foi realizada.

Recentemente, Lyan et al., ${ }^{12}$ avaliaram estratégias diferentes de ablação por cateter de taquicardia atrial local originando próximo à região do feixe de His em 68 pacientes, e concluíram que o índice de sucesso agudo de ablação de TA para-Hissiana pela $\mathrm{CNC}$ foi mais alto do que a ablação no septo do AE e no septo do AD ( $p<0,05)$. Por esse motivo, eles argumentam que a $\mathrm{CNC}$ deve ser a primeira abordagem e a preferencial para essas taquicardias, independentemente do tempo de ativação local. Esse também e o posicionamento de Bohora et al., ${ }^{13}$ Por outro lado, Madaffari et al., ${ }^{11}$ propõem que a CNC é uma entre três abordagens possíveis para alcançar o sucesso, e que a escolha deve ser baseada no momento de ativação local.
Concordamos com Lyan et al., ${ }^{12}$ e Bohora et al., ${ }^{13}$ já que a abordagem de $\mathrm{CNC}$ parece ser a primeira escolha para realizar a ablação nesse cenário, com altos índices de sucesso.

\section{Conclusão}

Este estudo confirma as observações prévias de que o mapeamento e a ablação por cateter de taquicardia atrial focal com ativação precoce próxima ao feixe de His, a partir da cúspide aórtica não coronária $(\mathrm{CNC})$ é um procedimento eficiente e aparentemente seguro. Acreditamos que a exploração retroaórtica deva ser obrigatória nesse caso. Um eletrocardiograma de superfície pode sugerir o alvo adequado próximo à região do feixe de His, mas não em todos os casos. $\mathrm{O}$ conhecimento das relações da CNC com o sistema de condução é fundamental na ablação dessas taquicardias. Esses achados devem ser considerados na estratégia terapêutica dessa arritmia complexa.

\section{Contribuição dos Autores}

Concepção e desenho da pesquisa: Chokr M, Moura LG, Sousa IBS, Scanavacca M; Obtenção de dados: Chokr M, Moura LG, Sousa IBS, Pisani CF, Hardy CA, Melo SL, Ponte Filho AD, Costa IP, Tavora RV, Sacilotto L, Wu TC, Darrieux FCC, Hachul DT, Aiello V, Scanavacca M; Análise e interpretação dos dados: Chokr M, Moura LG, Sousa IBS, Pisani CF, Ponte Filho AD, Costa IP, Sacilotto L, Wu TC, Darrieux FCC, Hachul DT, Aiello V, Scanavacca M; Análise estatística e Obtenção de financiamento: Chokr M, Moura LG, Sousa IBS; Redação do manuscrito: Chokr M, Moura LG, Sousa IBS, Aiello V, Scanavacca M; Revisão crítica do manuscrito quanto ao conteúdo intelectual importante: Chokr M, Moura LG, Sousa IBS, Pisani CF, Scanavacca M.

\section{Potencial conflito de interesses}

Declaro não haver conflito de interesses pertinentes.

\section{Fontes de financiamento}

O presente estudo não teve fontes de financiamento externas.

\section{Vinculação acadêmica}

Não há vinculação deste estudo a programas de pós-graduação.

\section{Aprovação ética e consentimento informado}

Este artigo não contém estudos com humanos ou animais realizados por nenhum dos autores.

\section{Errata}

Edição de Janeiro de 2021, vol. 116 (1), págs. 119-126

No Artigo Original "Ablação por Cateter de Taquicardia Atrial Focal com Ativação Precoce Próxima ao Feixe de His, a Partir da Cúspide Aórtica não Coronária“, com número de DOI: https://doi.org/10.36660/abc.20180449, publicado no periódico Arquivos Brasileiros de Cardiologia, 116(1):119-126, na página 119, corrigir o nome da autora Vera Aiello para Vera Demarchi Aiello. 


\section{Referências}

1. Tada H, NaitoS, Miyazaki A, OshimaS, Nogami A, Taniguchi K. Successful catheter ablation of atrial tachycardia originating near the atrioventricular node from the noncoronary sinus of Valsalva. Pacing Clin Electrophysiol. 2004; 27(10):1440-3.

2. Hasdemir C, Aktas S, Govsa F, Aktas E, Kocak A, Bozkaya Y, et al. Demonstration of ventricular myocardial extensions into the pulmonary artery and aorta beyond the ventriculo-arterial junction. Pacing Clin Electrophysiol. 2007;30(4):534-9.

3. Jongbloed MR, Mahtab EA, Blom NA, Schalij MJ. Gittenberger- de Groot AC. Development of the cardiac conduction system and the possible relation to predilection sites of arrhythmogenesis. Scient Word J. 2008;8:239-69.

4. Chen CC, Tai CT, Chiang CE, Yu WC, Lee SH, Chen YJ, et al. Atrial tachycardias originating from the atrial septum: electrophysiologic and radiofrequency ablation. J Cardiovasc Electrophysiol. 2000;11(7):744-9.

5. Chen M, Yang B, Wright M, Cabrera JA, Ju W, Chen H, et al. Successful catheter ablation of focal atrial tachycardia from the ascending aorta: a novel location and approach. Circ Arrhythm Electrophysiol. 2009;2(6):e34-e41.

6. Park J, Wi J, Joung B, Lee MH, Kim YH, Hwang C, et al. Prevalence, risk, and benefits of radiofrequency catheter ablation at the aortic cusp for treatment of mid to anteroseptal supra-ventricular tachyarrhythmias. Int J Cardiol. 2013; 167(3):981-6.

7. Sasaki T, Hachiya H, Hirao K, Higuchi K, Hayashi T, Furuwaka T, et al. Utility of distinctive local electrogram pattern and aortographic anatomical position in catheter manipulation at coronary cusps. J Cardiovasc Electrophysiol. 2011; 22(5):521-9.

8. Toniolo M, Rebellato L, Poli S, Daleffe E, Proclemer A. Efficacy and safety of catheter ablation of atrial tachycardia through a direct approach from noncoronary sinus of Valsalva. Am J Cardiol. 2016;118(12):1847-54.

9. Bitaraes B, ChokrM, Pisani C, Leite T, Avila W, Scanavacca M. Catheter ablation of atrial tachycardia on the non-coronary aortic cusp during pregnancy without fluoroscopy. HeartRhythm Case Rep. 2018 Dec; 4(12): 566-9.

10. Ouyang F, MA J, Ho SY, Bansch D, Schmidt B, Ernst S, et al. Focal atrial tachycardia originating from the non-coronary aortic sinus: electrophysiological characteristics and catheter ablation. J Am Coll Cardiol. 2006;48(1):122-31.

11. Madaffari A, Grosse A, Bruneli M, Frommhold M, Dahne T, Oreto G, et al. Eletrocardiographic and electrophysiological characteristics of atrial tachycardia with early activation close to the His-Bundle. J Cardiovasc Electrophysiol .2016;27(2):175-82.

12. Lyan E, Toniolo M, Tsyganov A, Rebellato L, Proclemer A, Manfrin M, et al. Compararison of strategies for catheter ablation of focal atrial tachycardia originating near His bundle region. Heart Rhythm. 2017;14(7):998-1005.

13. Bohora S, Lokhandwala Y, Sternick EB, Anderson RH, Wellens HJ. Reappraisal and new observations on atrial tachycardia ablated from the non-coronary aortic sinus of Valsalva. Europace. 2018;20(1):124-33. 